\title{
Prognostic Impact of CEACAM1 in Node-Negative Ovarian Cancer Patients
}

\author{
Leticia Oliveira-Ferrer $\mathbb{D}^{\mathrm{D}}{ }^{1}$ Roshni Goswami, ${ }^{1}$ Vladimir Galatenko, ${ }^{2}$ Yi Ding, \\ Kathrin Eylmann, ${ }^{1}$ Karen Legler, ${ }^{1}$ Sascha Kürti, ${ }^{1}$ Barbara Schmalfeldt, ${ }^{1}$ \\ and Karin Milde-Langosch ${ }^{1}$ \\ ${ }^{1}$ Department of Gynecology, University Medical Center Hamburg-Eppendorf, Martinistraße 52, 20246 Hamburg, Germany \\ ${ }^{2}$ Moscow State University, Moscow, Russia
}

Correspondence should be addressed to Leticia Oliveira-Ferrer; ferrer@uke.de

Received 9 April 2018; Accepted 31 May 2018; Published 28 June 2018

Academic Editor: Gad Rennert

Copyright (c) 2018 Leticia Oliveira-Ferrer et al. This is an open access article distributed under the Creative Commons Attribution License, which permits unrestricted use, distribution, and reproduction in any medium, provided the original work is properly cited.

\begin{abstract}
The underlying mechanisms of ovarian cancer (OvCa) dissemination are still poorly understood, and novel molecular markers for this cancer type are urgently needed. In search of adhesion molecules with prognostic relevance in OvCa, we compared tumors with good outcome (alive $>3$ years) and those with poor outcome ( $\mathrm{dead}<2$ years) within data from The Cancer Genome Atlas (TCGA). The carcinoembryonic antigen-related cell adhesion molecule 1 (CEACAM1) turned out as the only gene with differential expression in these groups. In order to further investigation on its role in OvCa, we analyzed CEACAM1 mRNA levels extracted from TCGA microarray data $(n=517)$ as well as CEACAM1 protein expression by Western blot analysis in a cohort of 242 tumor samples. Further, CEACAM1 localization in tumour tissue was evaluated by immunohistochemistry and CEACAM1 splice variants by RT-PCR in representative tumours. In Kaplan-Meier analysis, high CEACAM1 mRNA levels significantly correlated with longer survival $(p=0.008)$. By Western blot analysis in the second cohort, similar associations of high CEACAM1 protein levels with longer recurrence-free survival (RFS, $p=0.035)$ and overall survival $(\mathrm{OAS}, p=0.004)$ were observed. In multivariate Cox regression analysis including clinical prognostic parameters, CEACAM1 mRNA or protein expression turned out as independent prognostic markers. Stratified survival analysis showed that high CEACAM1 protein expression was prognostic in node-negative tumors $(p=0.045$ and $p=0.0002$ for DFS and OAS) but lost prognostic significance in node-positive carcinomas. Similarly, high CEACAM1 mRNA expression did not show prognostic relevance in tumors with lymphatic invasion (L1) but was associated with longer survival in cases without lymphovascular involvement. Further analysis showed a predominance of $4 \mathrm{~S}$ and $4 \mathrm{~L}$ isoforms and mostly membraneous CEACAM1 localization in ovarian tumours. Our results suggest that CEACAM1 might be an independent favorable prognostic marker in OvCa, especially in the subgroup of patients with solely intraperitoneal metastasis.
\end{abstract}

\section{Introduction}

Epithelial ovarian carcinoma (EOC) is the gynecologic tumor with the highest mortality. Since it is asymptomatic in early development, it is mostly diagnosed in advanced stages when tumor dissemination has already taken place. Tumor spread occurs either intraperitoneally, without the nodal involvement and/or through lymphatics, giving rise to retroperitoneal metastatic lesions. Up to now, the biologic background of these two different metastatic routes is poorly understood.
Independently of the dissemination mode, ovarian cancer therapy includes optimal surgical tumor reduction (debulking) followed by platin-based combination chemotherapy. In spite of intensive research, there are no established molecular prognostic or predictive markers for this cancer type, and new molecular targets for an individualized therapy are urgently needed.

The carcinoembryonic antigen-related cell adhesion molecule 1 (CEACAM1) is a member of the carcinoembryonic antigen (CEA) family and belongs to the immunoglobulin 
superfamily. This glycoprotein can bind homophilically as well as heterophilically to the other CEA family members [1]. Currently, 12 alternative splicing forms of the CEACAM1 gene are known $[2,3]$, differing in the number of extracellular immunoglobulin-like domains, the membrane anchorage and the length of the cytoplasmic domain [4]. Among them, only four isoforms are expressed at mRNA level: CEACAM1-4L, CEACAM1-4S, CEACAM1-3L, and CEACAM1-3S, including 4 and 3 heavily glycosylated extracellular domains and a long (L) or short (S) cytoplasmic tail, respectively [2]. CEACAM1 is expressed in the epithelia and leukocytes in addition to the endothelia of newly formed vessels and exerts very different biological functions such as immune response regulation, neovascularization or insulin clearance [5].

The role of CEACAM1 in cancer strongly differs depending on the origin of the tumor cell. Downregulation of CEACAM1 has been described in prostate, colon, and breast cancer, whereas CEACAM1 upregulation correlates with disease progression in melanoma and pulmonary adenocarcinoma [6-11]. Here, CEACAM1 is involved in several cellular functions such as proliferation, apoptosis, angiogenesis, invasion, and migration [12]. In selected tumor types like melanoma and glioma, first experiments indicate that CEACAM1 might be a suitable target for immunotherapy $[13,14]$. The majority of the mentioned studies analyzed total CEACAM1 levels. Recently, the specific role of concrete isoforms has been studied by different groups in melanoma and colon cancer [15-17].

In ovarian cancer, $\mathrm{Li}$ et al. have recently shown a membrane-associated CEACAM1 staining in primary lowgrade adenocarcinomas, whereas in high-grade adenocarcinomas and metastatic lesions CEACAM1 were mainly localized in the cytoplasm. These data suggest a tumor suppressor function of membranous CEACAM1, while cytoplasmic CEACAM1 might be involved in tumor progression and metastasis [18].

In order to deeply evaluate the relevance of CEACAM1 for ovarian cancer progression, we analyzed its predictive and prognostic value at both the mRNA and protein level in two well-characterized ovarian cancer cohorts.

\section{Material and Methods}

2.1. Patients. For Western blot analysis, a total of 242 patients with epithelial ovarian tumors and primary surgery at the University Medical Centre HamburgEppendorf between 1997 and 2012 were included. Patients gave their written approvals for examining tissue samples and reviewing their medical records according to our investigational review board and ethics committee guidelines (number 190504). The median follow-up time for patients with primary cancer $(n=210)$ was 21 months. Clinical outcome of all patients was followed from date of surgery until December 2016. Detailed patient characteristics are listed in the Supplementary Table S1. For comparison, samples from four benign ovarian cystadenomas, 16 tumors of low malignant potential (LMP, borderline tumors), and 12 recurrent carcinomas were analyzed.
2.2. Western Blot Analysis. Western blot analysis was performed as described previously $[19,20]$. Briefly, equal amounts of protein $(20 \mu \mathrm{g})$ of each sample were loaded per well. The protein lysate from the cell line OAW42 was used as a reference and internal control in all blots. After electrophoresis and blotting to PVDF membranes, CEACAM1 was detected using anti-human CEACAM-1/ CD66a antibody (AF2244, R\&D Systems, Minneapolis, USA) antibody. Membranes were blocked 1 hour at room temperature with $1 \%$ blocking solution containing $0.1 \mathrm{M}$ maleic acid and $0.15 \mathrm{~m} \mathrm{NaCl} \mathrm{pH7.5} \mathrm{in} \mathrm{TBST} \mathrm{and} \mathrm{subse-}$ quently incubated with the antibody $(0.2 \mu \mathrm{g} / \mathrm{ml}$ in $0.1 \%$ blocking/TBST solution) overnight at $4^{\circ} \mathrm{C}$. Secondary antibody (mouse anti Goat, sc-2354, Santa Cruz Biotechnology Inc., Heidelberg, Germany) was also diluted in $0.1 \%$ blocking solution and incubation was performed for 1 hour at room temperature. Equal loading was verified by immunoblotting with $\beta$-actin antibody (sc-47778, Santa Cruz Biotechnology). After visualization by chemiluminescence reagent (SuperSignal West Pico kit, Pierce, Rockford, IL, USA), band intensities were quantified by densitometry (Imaging Densitometer GS-700, Bio-Rad, Munich, Germany) and calculated as percent intensity of the specific control sample.

2.3. Immunohistochemistry. Immunohistochemical analyses were performed as previously described [19]. Briefly, for the detection of CEACAM1 on an ovarian cancer tissue, slides of $4 \mu \mathrm{m}$ were deparaffinized, microwaved in citrate buffer $\mathrm{pH} 6$, and incubated overnight at $4^{\circ} \mathrm{C}$ with the CD66a polyclonal antibody (R\&D Systems, Minnesota, USA; concentration: $2.5 \mu \mathrm{g} / \mathrm{ml}$ ). For detection, slides were incubated with biotin-labelled anti-goat immunoglobulin (IgG), preformed ABC-Complex (Vectastain, Vector Laboratories) and DABsubstrate kit (Vectastain, Vector Laboratories). All slides were counterstained with haematoxylin. As negative controls, normal goat immunoglobulin (Dako Denmarck A/S, Glostrup, Denmark) was used instead of primary antibody. Images were performed using an AxioVision40 Microscope (Carl Zeiss Imaging Solutions).

2.4. Isoform-Specific RT-PCR. RNA extraction and quality analyses were performed as mentioned before [21]. $1 \mu \mathrm{g}$ RNA was reverse transcribed using the Transcriptor First-Strand Synthesis Kit (Roche). The PCR were performed by ALLin ${ }^{\mathrm{TM}}$ Hot Start Taq Polymerase Kit (highQu, Kraichtal, Germany) in a total volume of $25 \mu \mathrm{l}$ containing $1 \mu \mathrm{l}$ of firststrand cDNA solution, $0.5 \mu \mathrm{l}$ of ALLin Hot Start Taq Polymerase $(5 \mathrm{u} / \mu \mathrm{l}), 5 \mu \mathrm{l}$ of $5 \mathrm{X}$-ALLin PCR Buffer, $2 \mu \mathrm{l}$ each of the PCR primers $(10 \mathrm{pmol} / \mu \mathrm{l})$, and $14.5 \mu \mathrm{l} \mathrm{H} \mathrm{H}_{2} \mathrm{O}$. The reactions were initiated by heating the samples to $95^{\circ} \mathrm{C}$ for $60 \mathrm{~s}$, followed by 40 cycles at $95^{\circ} \mathrm{C}$ for $15 \mathrm{~s}, 55-65^{\circ} \mathrm{C}$ for $15 \mathrm{~s}$, and $72^{\circ} \mathrm{C}$ for $15 \mathrm{~s}$ and an extension at $4^{\circ} \mathrm{C}$ for $10 \mathrm{~min}$. The products were analyzed on $2 \%$ agarose gels in Tris-borateEDTA buffer and visualized by ethidium bromide staining. The sense (forward (FP)) primer is common for the two isoforms, whereas the antisense (backward (BP)) primers are selective for the $\mathrm{L}$ or the $\mathrm{S}$ isoform, respectively. Following oligonucleotide, primers were used in order to amplify the 
different isoforms as previously described [22]: humanCEACAM1_FP49: 5'-GCAACAGGACCACAGTCAAGAC GA-3' ${ }^{\prime}$, humanCEACAM1_BP60: $5^{\prime}$-GTGGTTGGAGACTG AGGGTTTG-3' and humanCEACAM1_BP59: 5'-TGGA GTGGTCCTGAGCTGCCG-3' ${ }^{\prime}$. For quantification of CEACAM1-4L/4S ratio, a triple-primer PCR procedure was used as previously described [22].

2.5. Gene Expression Analysis and Statistics. Gene expression data from serous ovarian adenocarcinomas were retrieved from The Cancer Genome Atlas (TCGA) Research Network. Patient annotation data and CEL files with scans of microarrays (microarray type Affymetrix HT HG U133A) were downloaded from TCGA [23] and jointly preprocessed in Affymetrix Expression Console using the robust multiarray average (RMA) method [24]. In order to identify prognostic genes, a standard differential expression analysis was applied to the group of patients with survival less time than two years versus the group of patient with vital status "alive" and follow-up of at least three years. The analysis was limited to patients with stages III and IV, and the groups contained 97 and 71 patients, respectively. The differential expression analysis was performed in Affymetrix Transcriptome Analysis Console. As the compared groups were similar, relatively liberal thresholds were used, namely, 1.5x for fold change and 0.05 for $p$ value. Statistical analyses were performed using the Statistical Package for Social Sciences (SPSS) program (SPSS Inc., Chicago, IL, USA), version 15.

For statistical analysis of CEACAM1 protein expression levels in primary carcinomas, all tumor cases were divided into two groups of equal size $(</>$ median), representing low and high protein expression. Chi-square tests were used to examine the correlations between CEACAM1 and clinicopathologic factors (age, FIGO stage, histology subtype, grading, and residual postoperative tumor). For prognostic parameters, the following groups were compared: histological grading (G1/G2 versus G3), FIGO stage (I/II versus III versus IV), histological subtype (serous versus others), and residual tumor (none versus $>1 \mathrm{~cm}$ ). Survival curves were plotted by Kaplan-Meier analysis. Differences between survival curves were evaluated by log-rank tests. Probability values ( $p$ value) $\leq 0.05$ were considered statistically significant.

\section{Results}

3.1. CEACAM1 mRNA Levels in Ovarian Carcinomas (TCGA Cohort). Since we were interested in the role of adhesion proteins in ovarian cancer progression, we decided to analyze the microarray data of the TCGA cohort. For this purpose, 536 CEL files were downloaded. We excluded 16 duplicates and 3 cases with missing follow-up data, resulting in a cohort of 517 cases. The characteristics of this cohort are given in the Supplementary Table S2.

In the first screening approach, we compared stage III/IV cases with relatively good outcome (alive $>3$ years) and those with poor outcome (dead $<2$ years). Sixty-two probesets passed the thresholds, including only one adhesion molecule, CEACAM1 (Affymetrix number 209498_at), with a 1.5 fold higher expression in tumors with a better prognosis. This led us to a more intensive analysis of CEACAM1 expression in this cohort.

Among the expression data generated by Affymetrix chips, there are five probesets for CEACAM1 (Affymetrix number 209498_at; 211883_x_at; 2118899_x_at; 210610_at; 206576_s_at). The mean expression levels in this cohort were relatively low for all probesets, with highly significant correlations among each other (not shown). Therefore, we concentrated on the probeset 209498_at with differential expression between the two groups and the highest expression level among the probesets (mean expression 23.2; median 12.4; range 5 to 327). The sequences of the probes included in this probeset allow for the detection of all CEACAM1 mRNA variants (Affymetrix).

For further analysis, the cohort was divided into two groups with low or high CEACAM1 mRNA levels with the median as the cutoff value. By Chi-square tests, these groups were compared with respect to the known tumor parameters. There was no significant association of CEACAM1 expression with age at diagnosis, grading, clinical stage, residual disease after surgery, and lymphovascular invasion and vascular invasion (Supplementary Table S3).

In survival analysis, we could show a significantly longer survival in carcinomas with high CEACAM1 mRNA levels $(p=0.008$; Figure 1(a)). In stratified Kaplan-Meier analysis regarding the presence of lymphovascular invasion, CEACAM1 was weakly prognostic in L0 tumors $(p=0.079)$ but not in L1 carcinomas (Figure 1(b)), whereas no difference in tumors with and without vascular invasion was observed (not shown).

In multivariate Cox regression analysis also including clinical stage, residual disease after surgery and histological grading, high CEACAM1 mRNA levels (>median) turned out as an independent and significant prognostic indicator with a hazard ratio of 0.75 (95\% CI $0.59-0.95$; $p=0.019$; Table 1).

3.2. CEACAM1 Protein Expression in Ovarian Tumor Samples. In order to validate the prognostic significance of CEACAM1 expression on a protein level, Western blot analysis was performed in tissue samples from our clinic, including 210 primary ovarian carcinomas, 12 recurrent tumors, 16 borderline tumors, and 4 cystadenomas. Due to a variety of splice variants and glycosylation variants, the size of the detected CEACAM1 bands ranged from 70 to $200 \mathrm{kDa}$, with strong variations in band intensity (Figure 2(a)). The different variants did not always appear as clearly distinguishable bands in Western blots, but in many cases, it led to a continuous smear. For densitometry, all CEACAM1 bands were therefore combined, and band intensities were calculated relative to the positive control cell line OAW42 which was set as 1 and corrected for equal actin loading.

Regarding tumors of different malignancy, no significant differences in CEACAM1 expression between cystadenomas, borderline tumors, and invasive carcinomas were found. In addition, expression did not differ between primary and recurrent carcinomas (not shown). 


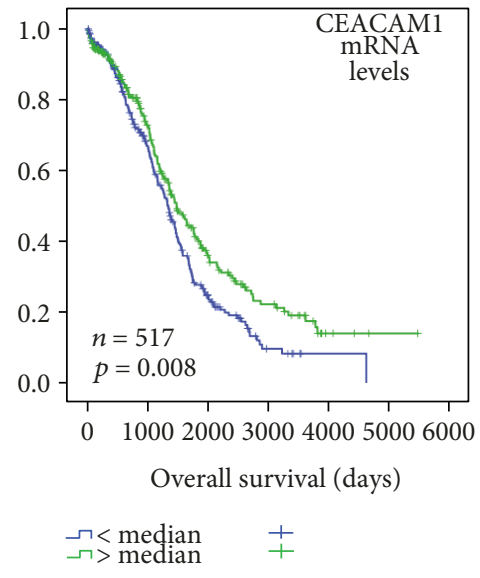

(a)

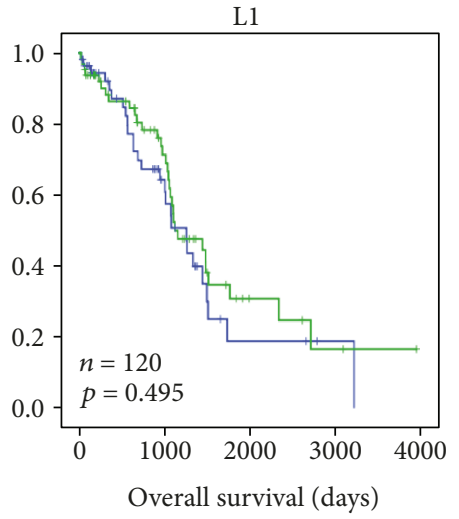

(b)

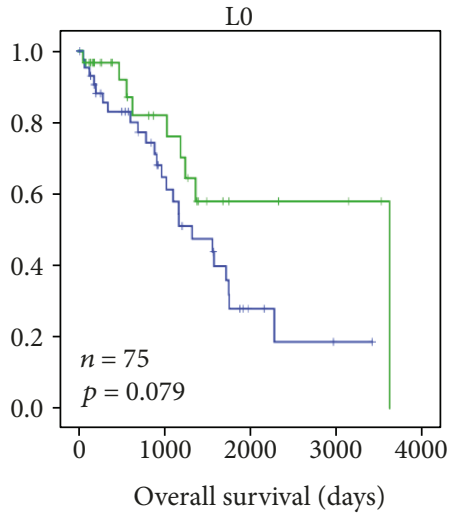

FiguRE 1: CEACAM1 mRNA levels in ovarian carcinomas (TCGA cohort). (a) Kaplan-Meier analysis showing a significant correlation of high CEACAM1 mRNA levels with longer overall survival. (b) Kaplan-Meier analysis and log-rank tests stratified for tumors with (L1) and without (L0) lymphovascular invasion. High CEACAM1 mRNA levels showed a clear association $(p=0.079)$ with shorter overall survival only in patients without lymphovascular invasion (L0) in comparison with those with lymphovascular invasion (L1).

TABle 1: Multivariate Cox regression analysis including CEACAM1 RNA levels.

\begin{tabular}{|c|c|c|c|c|}
\hline \multicolumn{5}{|c|}{ Overall survival } \\
\hline Variables in multivariate analysis & & $p$ & HR & $95 \% \mathrm{CI}$ \\
\hline CEACAM1 mRNA level & $>$ median & 0.019 & 0.75 & $0.59-0.95$ \\
\hline \multirow{3}{*}{ Clinical stage } & I-II & 0.136 & & \\
\hline & III & 0.183 & 1.65 & $0.79-3.44$ \\
\hline & IV & 0.007 & 2.05 & $0.94-4.43$ \\
\hline Tumor grading & G3 & 0.127 & 1.32 & $0.92-1.89$ \\
\hline \multirow{4}{*}{ Residual tumour after surgery } & No macroscopic tumour & 0.0002 & & \\
\hline & $1-10 \mathrm{~mm}$ & 0.001 & 1.87 & $1.31-2.68$ \\
\hline & $11-20 \mathrm{~mm}$ & 0.004 & 2.26 & $1.31-3.92$ \\
\hline & $>20 \mathrm{~mm}$ & 0.00001 & 2.51 & $1.67-3.77$ \\
\hline
\end{tabular}

Regarding the primary ovarian carcinoma samples, all cases were divided into two groups with CEACAM1 expression below/above the median value for statistical analysis. Using these groups, we found significant correlations of high CEACAM1 expression with the presence of distant metastasis, but no significant associations with grading, residual tumor after surgery, and lymph node involvement. Regarding FIGO stage, significantly higher CEACAM1 levels were detected in early stages (FIGO I-IIIb) and cases with distant metastasis (FIGO IV) in comparison to the most frequent stage IIIc tumors (Table 2). Pearson correlation did not reveal any association of CEACAM1 expression with age at diagnosis or CA125 serum levels before surgery (not shown).

Kaplan-Meier analysis revealed a significant association of high CEACAM1 levels with a longer recurrencefree survival $(p=0.035)$ and overall survival $(p=0.004$; Figure 2(b)) indicating that CEACAM1 might function as a tumor suppressor in ovarian carcinomas. Hazard ratios in tumors with high CEACAM1 expression were $0.516(95 \%$ CI $0.326-0.817 ; p=0.005)$ for death and 0.669 (95\% CI $0.457-0.980 ; p=0.039$ ) for recurrence.
In stratified Kaplan-Meier analysis regarding the lymph node involvement, high CEACAM1 expression was strongly prognostic of longer RFS and OAS in node-negative tumors, whereas it lost prognostic significance in node-positive carcinomas (Figure 2(c) and 2(d)). In multivariate Cox regression analysis including clinical stage, residual tumor after surgery, and nodal involvement, CEACAM1 remained a prognostic indicator for overall survival but lost its significance for recurrence-free survival (Table 3 ).

3.3. CEACAM1 Localization in Ovarian Cancer by Immunohistochemistry. In order to validate that CEACAM1 protein is expressed in tumor cells, we performed immunohistochemistry with formalin-fixed, paraffin-embedded tumor samples $(n=15)$ from the Western blot cohort. Regarding tumor cells, different staining patterns were observed: The most frequent type included membranous CEACAM1 staining, mainly within lumen-like structures of the tumor, partly accompanied by weak cytoplasmic reactivity (Figures 3(a)-3(c)). In some cases, there was a strong cytoplasmic staining in $<20 \%$ of the tumor cells 

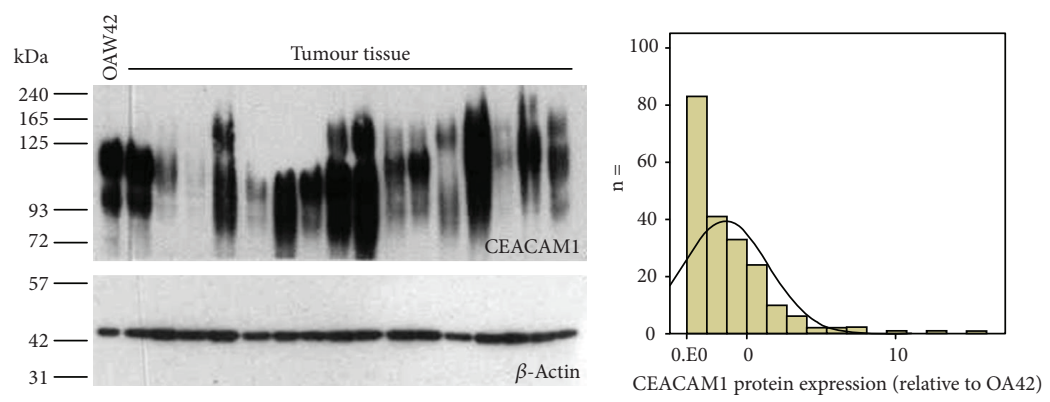

(a)
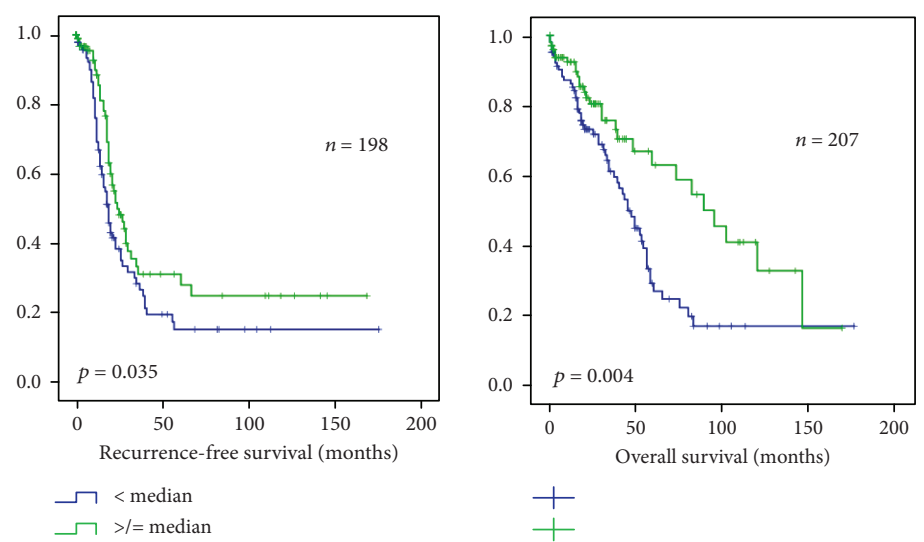

(b)
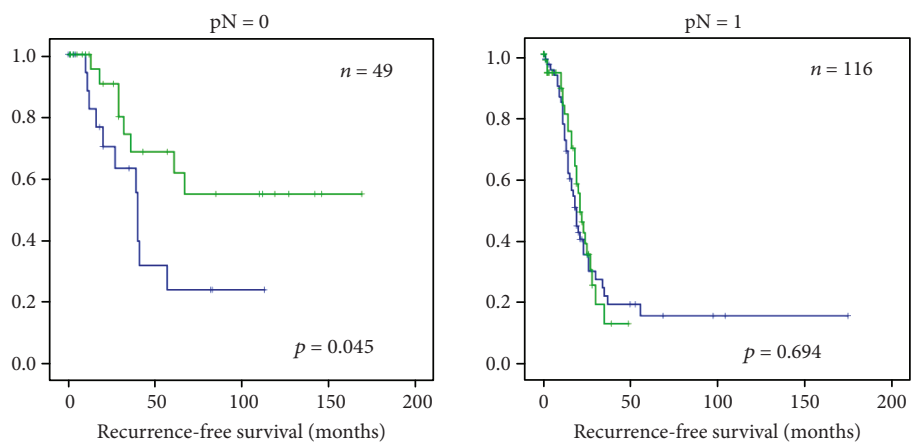

(c)
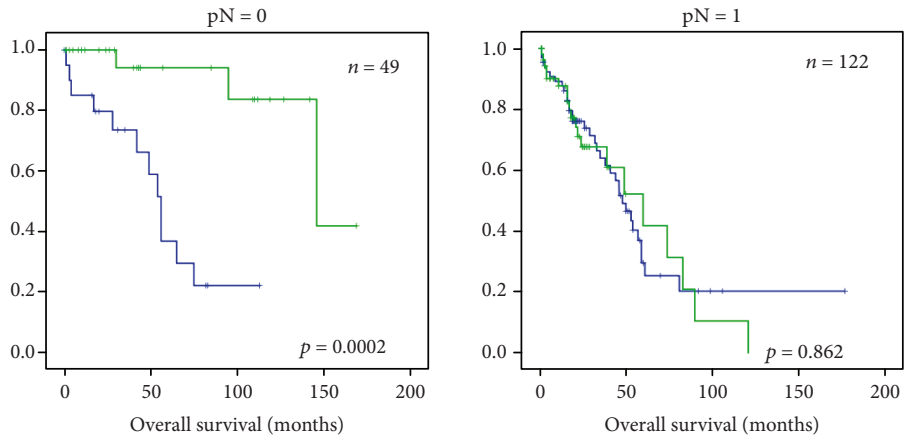

(d)

FIGURE 2: CEACAM1 protein expression in clinical tumor tissue samples. (a) Representative Western blot results of CEACAM1 expression in ovarian carcinomas (left) and distribution of the relative expression within the cohort (right). Protein extract from the ovarian cancer cell lines OAW42 was included in each gel as an internal control. (b) Kaplan-Meier analysis showing correlations of high CEACAM1 protein expression with longer recurrence-free survival and overall survival. (c, d) Kaplan-Meier analysis and log-rank tests, stratified for tumors with $(\mathrm{pN}=1)$ and without $(\mathrm{pN}=0)$ nodal involvement. High CEACAM1 expression correlated significantly with shorter recurrence-free survival and overall survival only in patients without nodal involvement. 
TABLE 2: Correlations of CEACAM1 protein expression with clinical and histological tumor parameters (missing values to $n=210$ : no information).

\begin{tabular}{|c|c|c|c|c|}
\hline & & $\begin{array}{c}\text { Low CEACAM1 expression } \\
\text { <median }\end{array}$ & $\begin{array}{l}\text { High CEACAM1 expression } \\
\text { >median }\end{array}$ & $p$ \\
\hline \multirow{2}{*}{ Clinical stage } & FIGO Ia-IIIb & 4 & 14 & \\
\hline & FIGO IIIc-IV & 97 & 86 & 0.013 \\
\hline \multirow{2}{*}{ Grading } & G1-2 & 29 & 26 & \\
\hline & G3 & 75 & 74 & 0.762 \\
\hline \multirow{2}{*}{ Lymph node involvement } & N0 & 21 & 28 & \\
\hline & N1 & 67 & 57 & 0.185 \\
\hline \multirow{2}{*}{ Distant metastasis } & M0 & 79 & 63 & \\
\hline & M1 & 16 & 27 & 0.034 \\
\hline \multirow{3}{*}{ Residual tumor after surgery } & No macroscopically visible tumor & 67 & 71 & \\
\hline & $>1 \mathrm{~cm}$ & 24 & 14 & \\
\hline & $>1 \mathrm{~cm}$ & 13 & 11 & 0.273 \\
\hline
\end{tabular}

TABLE 3: Multivariate Cox regression analysis including CEACAM1 protein expression.

\begin{tabular}{|c|c|c|c|c|}
\hline \multicolumn{5}{|c|}{ Overall survival } \\
\hline Variables in multivariate analysis & & $p$ & HR & $95 \% \mathrm{CI}$ \\
\hline \multirow[t]{2}{*}{ CEACAM1 expression $</>$ median } & & 0.002 & 0.44 & $0.26-0.74$ \\
\hline & Ia-IIIb & 0.0001 & & \\
\hline \multirow[t]{3}{*}{ FIGO stage } & IIIc & 0.238 & 1.81 & $0.67-4.88$ \\
\hline & IV & 0.002 & 5.15 & $1.87-14.21$ \\
\hline & No macroscopic tumor & 0.0004 & & \\
\hline \multirow[t]{2}{*}{ Residual tumor after surgery } & $<1 \mathrm{~cm}$ & 0.002 & 2.33 & $1.37-3.97$ \\
\hline & $>1 \mathrm{~cm}$ & 0.002 & 2.90 & $1.47-5.71$ \\
\hline \multicolumn{5}{|c|}{ Recurrence-free survival } \\
\hline \multirow[t]{2}{*}{ CEACAM1 expression $</>$ median } & & 0.291 & 0.80 & $0.53-1.21$ \\
\hline & Ia-IIIb & 0.001 & & \\
\hline \multirow[t]{3}{*}{ FIGO stage } & IIIc & 0.005 & 4.55 & $1.59-13.04$ \\
\hline & IV & 0.0003 & 7.67 & $2.55-23.05$ \\
\hline & No macroscopic tumor & 0.0001 & & \\
\hline \multirow[t]{2}{*}{ Residual tumor after surgery } & $<1 \mathrm{~cm}$ & 0.005 & 2.04 & $1.25-3.34$ \\
\hline & $>1 \mathrm{~cm}$ & 0.0001 & 3.60 & $1.89-6.88$ \\
\hline
\end{tabular}

(Figure 3(d)), whereas in other cases, a weak or no CEACAM1 expression within tumor cells was detectable (Figures 3(e) and 3(f)). Regarding the stromal components, CEACAM1 staining was frequently found in lymphocytes (Figure 3(e)) and vessel walls (Figure 3(f)).

3.4. mRNA Levels of CEACAM1 Isoforms in Ovarian Cancer. The mRNA levels of the four isoforms CEACAM1-4L/4S/-3L and -3S were determined by RT-PCR using specific primers that discriminate between these splice variants as previously described [22]. Here, the forward primer is common for the two isoforms CEACAM1-4 and -3, whereas the backward primers are specific for the $\mathrm{L}$ or the $\mathrm{S}$ isoforms, respectively. Using this PCR procedure in a representative group of 13 samples, we found that ovarian cancer tissue expresses primarily both $4 \mathrm{~L}$ and the $4 \mathrm{~S}$ isoforms of CEACAM1, whereas a very weak or no detection of the CEACAM1-3L and -3S variants was observed
(Figure 3(g)). A triple-primer RT-PCR was performed to quantify the expression ratios of the $\mathrm{L}$ and $\mathrm{S}$ splice isoforms. In almost all analyzed tumor tissue samples, the $\mathrm{S}$ isoform was stronger expressed than the $\mathrm{L}$ isoform with a $\mathrm{S}: \mathrm{L}$ ratio from 1 to $5.5(3 \mathrm{~h})$.

\section{Discussion}

CEACAM1 is an immunoglobulin-like cell adhesion molecule with a broad range of biological functions that has been frequently described to play a key role during tumor progression in diverse tumor entities. Depending on the origin of the tumor, CEACAM1 might differently affect the tumorigenic process by acting as a tumor suppressor gene or as a metastatic driver. The present data show for the first time a significant association of CEACAM1 expression with disease outcome in ovarian cancer. This could be shown in two 


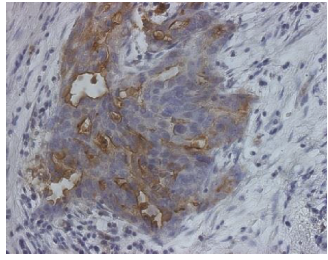

(a)

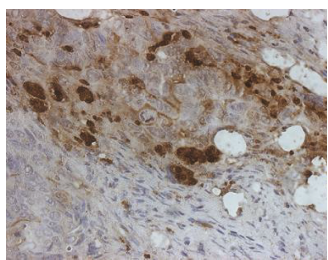

(d)

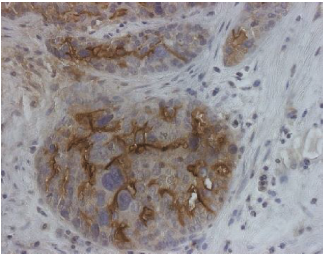

(b)

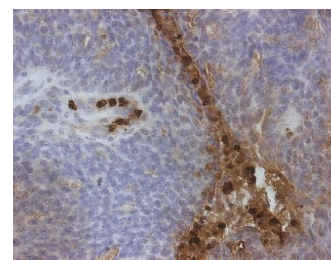

(e)

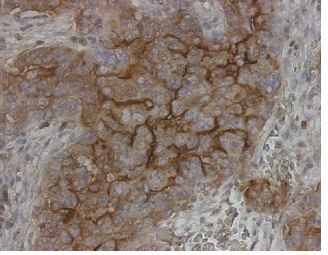

(c)

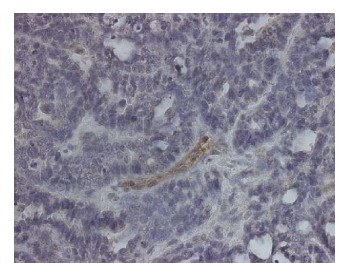

(f)

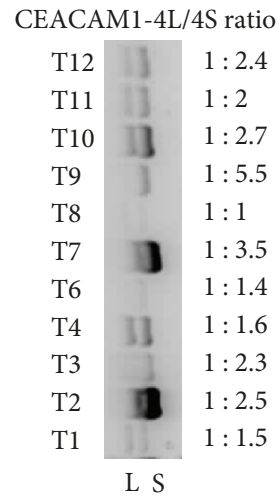

(h)

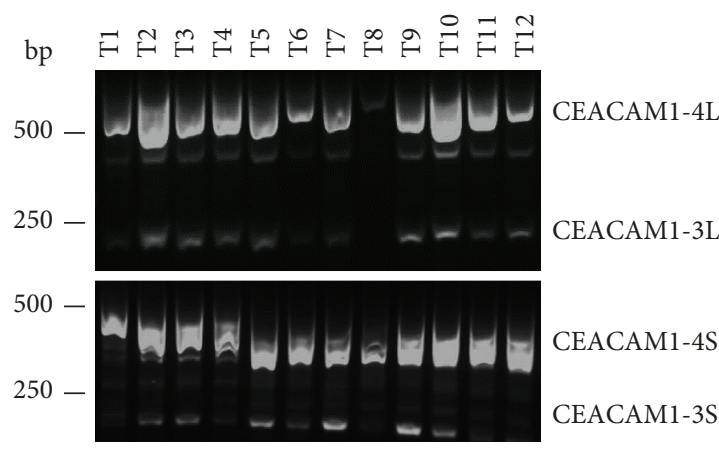

(g)

FIGURE 3: CEACAM1 protein localization and mRNA isoforms in selected ovarian tumors as detected by immunohistochemistry (a-f) and RT-PCR (g, h). CEACAM1 expression was observed in tumor cells as a mixed pattern of strong membranous staining, mainly within lumenlike structures and a weak cytoplasmic reactivity $(\mathrm{a}-\mathrm{c})$. Occasionally, a strong cytoplasmic CEACAM1 expression was observed in single cells or small cell aggregates (d). CEACAM1 staining was also found in lymphocytes (e) and endothelial cells from diverse small tumor-associated capillaries (f). RT-PCR of RNA isolated from ovarian cancer tissue using the oligonucleotides FP49 and BP60 (L-isoforms, upper panel) and FP49 and BP60 (S-isoforms, lower panel). Mainly products corresponding to the 4 (4L and 4S) isoform were amplified, whereas products corresponding to the 3 isoform were very weakly or not detected (g). A triple-primer RT-PCR was performed to quantify the expression ratios of the $\mathrm{L}$ and $\mathrm{S}$ splice isoforms. In all analyzed tumor tissues, the $\mathrm{S}$ isoform was expressed to a higher extent than the $\mathrm{L}$ isoform (h).

independent large cohorts on mRNA (TCGA data) and protein level (Hamburg cohort).

CEACAM1 has been found to be normally expressed in diverse epithelia such as in the colon, gallbladder, pancreas, kidney, prostate, and endometrium, frequently showing a characteristic apical membranous staining [25]. In contrast, in normal fallopian tube epithelium, which is considered to be the precursor tissue of most high-grade ovarian carcinomas, CEACAM1 expression was not detectable [26]. Regarding CEACAM1 deregulation during tumor progression, CEACAM1 is considered to be downregulated in breast, colon, endometrium, prostate, and hepatocellular carcinomas, suggesting a tumor suppressor role of this molecule, but it is upregulated in gastric carcinoma, squamous cell carcinoma of the lung, or melanoma [27]. Here, increased expression frequently correlates with metastasis and poor prognosis $[10,28,29]$. Our results show a strong CEACAM1 expression in most ovarian cancer tissue samples, whereas its impact on prognosis points to a tumor suppressor function. High CEACAM1 expression significantly correlates with longer recurrence-free and overall survival as shown at mRNA and protein levels in two independent cohorts comprising material from 517 and 210 ovarian cancer patients, respectively.

Controversial findings have also been reported about CEACAM1 function in tumor biology. As a tumor suppressor, its loss promotes early tumor development by reducing CEACAM1-mediated growth inhibitory signaling [30]. The $\mathrm{N}$-terminal domain of CEACAM1, essential for intercellular adhesion, was not necessary for this tumor inhibitory effect. An antiproliferative effect of CEACAM1 has also been shown in preclinical models in bladder, prostate, and colon cancer cell lines [31-34]. On the other hand, CEACAM1 has been described as a driver of invasion and metastasis in colorectal cancer and hepatocellular carcinoma $[35,36]$. Our data on two independent OvCa cohorts indicate that high CEACAM1 levels exert a tumor suppressor function in ovarian cancer, possibly by reducing tumor cell proliferation as 
previously described in other entities. In contrast, the described proinvasive and promigratory function of CEACAM1 seems to be not as relevant in ovarian cancer. Indeed, for intraperitoneal metastasis, which is the main route of tumor spread in those patients without nodal involvement, the key features for tumor progression are tumor cell growth, detachment, and immune evasion rather than cell motility and invasive potential.

Another functional aspect is the role of CEACAM1 as an immune modulator. In the context of cancer, there are contradictory reports describing CEACAM1 as an activator or repressor of the immune response [13]. Recently, a CEACAM1-associated decrease of STAT3 activity and CCL2 secretion was found in colorectal carcinoma, thus regulating inflammatory signalling networks and decreasing metastatic burden [37]. Further, CEACAM1-3S enhanced immunogenicity of melanoma cells by cell surface upregulation of NKG2D receptor ligands, thereby sensitizing them to lysis by natural killer cells [15]. In line with these studies, our data suggest that CEACAM1 overexpression might increase OvCa cell immunogenicity, thus decreasing their tumorigenicity.

Tumor spread in ovarian cancer occurs either intraperitoneally or through lymphatics, giving rise to retroperitoneal metastatic lesions. Remarkably, the correlation between high CEACAM1 protein levels and longer recurrence-free or overall survival was highly significant only within the subpopulation of ovarian cancer patients with solely intraperitoneal metastasis without nodal involvement. In contrast, CEACAM1 does not show any prognostic significance in tumours with nodal involvement in our Western blot cohort. The mRNA results obtained using the TCGA database which includes information about lymphovascular involvement, but not about nodal metastasis, point to the same direction. Thus, CEACAM1 expression might have an inhibitory impact on intraperitoneal metastasis but obviously loses this effect if lymphatic involvement takes place. The fact that a significant prognostic impact is shown in the entire cohort $(n=517)$, but only a borderline significance in the L0 and no impact in the L1 subcohorts can be explained by the relatively low case numbers with information about this point $(n=120 / n=75$ for L1/L0; Table S2).

Our data on the prognostic impact of CEACAM1 expression support the theory that these two modes of OvCa progression represent different tumor types which rely on different biologic backgrounds. Recent studies have shown that the tumor cell differentiation status (epithelial-mesenchymal transition) might influence the route of metastasis in ovarian cancer [38]. Accordingly, we could demonstrate that a $85 \mathrm{kDa}$ fragment of the epithelial marker E-Cadherin is associated with intraperitoneal metastasis, whereas VEGF-C and VEGF-D are more highly expressed in tumors displaying retroperitoneal tumor spread $[39,40]$. Although CEACAM1 expression does not correlate with the mode of OvCa metastasis, its relevance for patient outcome strongly differs between both ovarian cancer types.

Originally, most studies analyzed total CEACAM1 expression without taking into consideration the prevalence and specific role of certain isoforms. Indeed, recent results demonstrate an isoform-specific functionality of CEACAM1, thus increasing the complexity of this system. In malignant melanoma cell lines, CEACAM1-3S, CEACAM1-3L, CEACAM1-4S, and CEACAM1-4L affected migration, invasion, and immunogenicity in an isoform-specific manner [15]. Additionally, isoform-specific cellular localizations could be shown: CEACAM1-4 variants were mainly membrane associated, whereas CEACAM1-3 isoforms were predominantly localized intracellularly [15]. In our analysis of these isoforms in OvCa tumor tissue samples, we found high mRNA levels for CEACAM1-4L and -4 S, whereas CEACAM1-3L and $3 S$ were only weakly or not detectable. In line with prior results [15], we observed a primarily membranous CEACAM1 staining in most analyzed ovarian cancer samples together with a weak cytoplasmic expression. Further, in a small group of ovarian cancer samples, higher levels of the $4 \mathrm{~S}$ isoform compared to the long variant $4 \mathrm{~L}$ were found. The CEACAM1-4S isoform has been shown in breast cancer to inhibit tumor cell invasion and migration and to promote apoptosis [41, 42]. Further functional experiments would be necessary to specifically clarify the impact of both isoforms in ovarian cancer progression.

\section{Conclusion}

To our knowledge, this is the first study reporting a significant association of high CEACAM1 expression with a better outcome of ovarian cancer patients in two independent cohorts. This prognostic impact is restricted to tumors with intraperitoneal metastasis but without lymph node involvement. Thus, CEACAM1 might be an independent favorable prognostic or predictive marker for node-negative ovarian cancer patients.

\section{Data Availability}

The mRNA data used to support the findings of this study and corresponding clinical and histological information can be downloaded from The Cancer Genome Atlas (TCGA) Research Network [23]. The exact Western blot data used to support the findings of this study are available from the corresponding author upon request.

\section{Disclosure}

Vladimir Galatenko present address is Tauber Bioinformatics Research Center, University of Haifa, Haifa, Israel.

\section{Conflicts of Interest}

The authors declare that there is no conflict of interest regarding the publication of this paper.

\section{Acknowledgments}

The authors thank Maila Rossberg for excellent technical assistance. This work was funded by financial resources of the Clinic of Gynecology, University Medical Centre Hamburg-Eppendorf. 


\section{Supplementary Materials}

Table S1: cohort of ovarian cancer patients used for Western blot analysis. Table S2: TCGA cohort $(n=517)$ used for analysis of mRNA data. Table S3: correlations of CEACAM1 mRNA levels with clinical and histological tumour parameters. (Supplementary Materials)

\section{References}

[1] M. Rojas, A. Fuks, and C. P. Stanners, "Biliary glycoprotein, a member of the immunoglobulin supergene family, functions in vitro as a $\mathrm{Ca} 2(+)$-dependent intercellular adhesion molecule," Cell Growth \& Differentiation, vol. 1, no. 11, pp. 527-533, 1990.

[2] T. R. Barnett, L. Drake, and W. Pickle 2nd, "Human biliary glycoprotein gene: characterization of a family of novel alternatively spliced RNAs and their expressed proteins," Molecular and Cellular Biology, vol. 13, no. 2, pp. 1273-1282, 1993.

[3] M. Kuroki, F. Arakawa, Y. Matsuo, S. Oikawa, H. Nakazato, and Y. Matsuoka, "Three novel molecular forms of biliary glycoprotein deduced from cDNA clones from a human leukocyte library," Biochemical and Biophysical Research Communications, vol. 176, no. 2, pp. 578-585, 1991.

[4] S. D. Gray-Owen and R. S. Blumberg, "CEACAM1: contactdependent control of immunity," Nature Reviews. Immunology, vol. 6, no. 6, pp. 433-446, 2006.

[5] K. Kuespert, S. Pils, and C. R. Hauck, "CEACAMs: their role in physiology and pathophysiology," Current Opinion in Cell Biology, vol. 18, no. 5, pp. 565-571, 2006.

[6] A. Ebrahimnejad, T. Streichert, P. Nollau et al., "CEACAM1 enhances invasion and migration of melanocytic and melanoma cells," The American Journal of Pathology, vol. 165, no. 5, pp. 1781-1787, 2004.

[7] W. Luo, M. Tapolsky, K. Earley et al., "Tumor-suppressive activity of CD66a in prostate cancer," Cancer Gene Therapy, vol. 6, no. 4, pp. 313-321, 1999.

[8] M. Neumaier, S. Paululat, A. Chan, P. Matthaes, and C. Wagener, "Biliary glycoprotein, a potential human cell adhesion molecule, is down-regulated in colorectal carcinomas," Proceedings of the National Academy of Sciences of the United States of America, vol. 90, no. 22, pp. 10744-10748, 1993.

[9] L. Riethdorf, B. W. Lisboa, U. Henkel, M. Naumann, C. Wagener, and T. Löning, "Differential expression of CD66a (BGP), a cell adhesion molecule of the carcinoembryonic antigen family, in benign, premalignant, and malignant lesions of the human mammary gland," The Journal of Histochemistry and Cytochemistry, vol. 45, no. 7, pp. 957-963, 1997.

[10] A. Thies, A. Berlin, G. Brunner et al., "Glycoconjugate profiling of primary melanoma and its sentinel node and distant metastases: implications for diagnosis and pathophysiology of metastases," Cancer Letters, vol. 248, no. 1, pp. 68-80, 2007.

[11] I. Thom, O. Schult-Kronefeld, I. Burkholder et al., "Expression of CEACAM-1 in pulmonary adenocarcinomas and their metastases," Anticancer Research, vol. 29, no. 1, pp. 249-254, 2009.

[12] N. Beauchemin and A. Arabzadeh, "Carcinoembryonic antigen-related cell adhesion molecules (CEACAMs) in cancer progression and metastasis," Cancer Metastasis Reviews, vol. 32, no. 3-4, pp. 643-671, 2013.
[13] M. Dankner, S. D. Gray-Owen, Y. H. Huang, R. S. Blumberg, and N. Beauchemin, "CEACAM1 as a multi-purpose target for cancer immunotherapy," OncoImmunology, vol. 6, no. 7, 2017.

[14] R. Ortenberg, Y. Sapir, L. Raz et al., "Novel immunotherapy for malignant melanoma with a monoclonal antibody that blocks CEACAM1 homophilic interactions," Molecular Cancer Therapeutics, vol. 11, no. 6, pp. 1300-1310, 2012.

[15] N. Ullrich, A. Heinemann, E. Nilewski et al., "CEACAM1-3S drives melanoma cells into NK cell-mediated cytolysis and enhances patient survival," Cancer Research, vol. 75, no. 9, pp. 1897-1907, 2015.

[16] S. Löffek, N. Ullrich, A. Görgens et al., "CEACAM1-4L promotes anchorage-independent growth in melanoma," Frontiers in Oncology, vol. 5, 2015.

[17] N. Yamamoto, S. Yokoyama, J. Ieda et al., "CEACAM1 and hollow spheroid formation modulate the chemosensitivity of colorectal cancer to 5-fluorouracil," Cancer Chemotherapy and Pharmacology, vol. 75, no. 2, pp. 421-430, 2015.

[18] N. Li, J. Y. Yang, X. Y. Wang, H. T. Wang, B. X. Guan, and C. J. Zhou, "Carcinoembryonic antigen-related cell adhesion molecule 1 is expressed and as a function histotype in ovarian tumors," Annals of Diagnostic Pathology, vol. 20, pp. 7-12, 2016.

[19] K. Eckhoff, R. Flurschutz, F. Trillsch, S. Mahner, F. Janicke, and K. Milde-Langosch, "The prognostic significance of Jun transcription factors in ovarian cancer," Journal of Cancer Research and Clinical Oncology, vol. 139, no. 10, pp. 1673$1680,2013$.

[20] S. Mahner, C. Baasch, J. Schwarz et al., "C-Fos expression is a molecular predictor of progression and survival in epithelial ovarian carcinoma," British Journal of Cancer, vol. 99, no. 8, pp. 1269-1275, 2008.

[21] L. Oliveira-Ferrer, A. Hessling, F. Trillsch, S. Mahner, and K. Milde-Langosch, "Prognostic impact of chondroitin-4sulfotransferase CHST11 in ovarian cancer," Tumour Biology, vol. 36, no. 11, pp. 9023-9030, 2015.

[22] B. B. Singer, I. Scheffrahn, R. Heymann, K. Sigmundsson, R. Kammerer, and B. Obrink, "Carcinoembryonic antigenrelated cell adhesion molecule 1 expression and signaling in human, mouse, and rat leukocytes: evidence for replacement of the short cytoplasmic domain isoform by glycosylphosphatidylinositol-linked proteins in human leukocytes," Journal of Immunology, vol. 168, no. 10, pp. 5139-5146, 2002.

[23] The Cancer Genome Atlas Research Network, "Integrated genomic analyses of ovarian carcinoma," Nature, vol. 474, pp. 609-615, 2011.

[24] R. A. Irizarry, B. Hobbs, F. Collin et al., "Exploration, normalization, and summaries of high density oligonucleotide array probe level data," Biostatistics, vol. 4, no. 2, pp. 249-264, 2003.

[25] F. Prall, P. Nollau, M. Neumaier et al., "CD66a (BGP), an adhesion molecule of the carcinoembryonic antigen family, is expressed in epithelium, endothelium, and myeloid cells in a wide range of normal human tissues," The Journal of Histochemistry and Cytochemistry, vol. 44, no. 1, pp. 35-41, 1996.

[26] K. V. Swanson, G. A. Jarvis, G. F. Brooks, B. J. Barham, M. D. Cooper, and J. M. L. Griffiss, "CEACAM is not necessary for Neisseria gonorrhoeae to adhere to and invade female genital epithelial cells," Cellular Microbiology, vol. 3, no. 10, pp. 681691, 2001. 
[27] S. Hammarstrom, "The carcinoembryonic antigen (CEA) family: structures, suggested functions and expression in normal and malignant tissues," Seminars in Cancer Biology, vol. 9, no. 2, pp. 67-81, 1999.

[28] E. Laack, H. Nikbakht, A. Peters et al., "Expression of CEACAM1 in adenocarcinoma of the lung: a factor of independent prognostic significance," Journal of Clinical Oncology, vol. 20, no. 21, pp. 4279-4284, 2002.

[29] S. Sivan, F. Suzan, O. Rona et al., "Serum CEACAM1 correlates with disease progression and survival in malignant melanoma patients," Clinical \& Developmental Immunology, vol. 2012, article 290536, 8 pages, 2012.

[30] L. Izzi, C. Turbide, C. Houde, T. Kunath, and N. Beauchemin, "Cis-determinants in the cytoplasmic domain of CEACAM1 responsible for its tumor inhibitory function," Oncogene, vol. 18 , no. 40 , pp. 5563-5572, 1999.

[31] J. T. Hsieh, W. Luo, W. Song et al., "Tumor suppressive role of an androgen-regulated epithelial cell adhesion molecule $(\mathrm{C}-\mathrm{CAM})$ in prostate carcinoma cell revealed by sense and antisense approaches," Cancer Research, vol. 55, no. 1, pp. 190-197, 1995.

[32] D. I. Kleinerman, C. P. Dinney, W. W. Zhang, S. H. Lin, N. T. Van, and J. T. Hsieh, "Suppression of human bladder cancer growth by increased expression of C-CAM1 gene in an orthotopic model," Cancer Research, vol. 56, no. 15, pp. 3431-3435, 1996.

[33] D. I. Kleinerman, P. Troncoso, S. H. Lin et al., "Consistent expression of an epithelial cell adhesion molecule (C-CAM) during human prostate development and loss of expression in prostate cancer: implication as a tumor suppressor," Cancer Research, vol. 55, no. 6, pp. 1215-1220, 1995.

[34] N. Leung, C. Turbide, B. Balachandra, V. Marcus, and N. Beauchemin, "Intestinal tumor progression is promoted by decreased apoptosis and dysregulated Wnt signaling in Ceacam1-/- mice," Oncogene, vol. 27, no. 36, pp. 49434953, 2008.

[35] J. Ieda, S. Yokoyama, K. Tamura et al., "Re-expression of CEACAM1 long cytoplasmic domain isoform is associated with invasion and migration of colorectal cancer," International Journal of Cancer, vol. 129, no. 6, pp. 1351-1361, 2011.

[36] S. Kiriyama, S. Yokoyama, M. Ueno et al., "CEACAM1 long cytoplasmic domain isoform is associated with invasion and recurrence of hepatocellular carcinoma," Annals of Surgical Oncology, vol. 21, Supplement 4, pp. 505-514, 2014.

[37] A. Arabzadeh, J. Dupaul-Chicoine, V. Breton et al., "Carcinoembryonic antigen cell adhesion molecule 1 long isoform modulates malignancy of poorly differentiated colon cancer cells," Gut, vol. 65, no. 5, pp. 821-829, 2016.

[38] K. Auer, A. Bachmayr-Heyda, S. Aust et al., "Peritoneal tumor spread in serous ovarian cancer-epithelial mesenchymal status and outcome," Oncotarget, vol. 6, no. 19, pp. 17261-17275, 2015.

[39] S. Kuerti, L. Oliveira-Ferrer, K. Milde-Langosch et al., "VEGF$\mathrm{C}$ expression attributes the risk for lymphatic metastases to ovarian cancer patients," Oncotarget, vol. 8, no. 26, pp. 43218-43227, 2017.

[40] F. Trillsch, S. Kuerti, C. Eulenburg et al., "E-cadherin fragments as potential mediators for peritoneal metastasis in advanced epithelial ovarian cancer," British Journal of Cancer, vol. 114, no. 2, pp. 213-220, 2016.
[41] J. Kirshner, C. J. Chen, P. Liu, J. Huang, and J. E. Shively, "CEACAM1-4S, a cell-cell adhesion molecule, mediates apoptosis and reverts mammary carcinoma cells to a normal morphogenic phenotype in a 3D culture," Proceedings of the National Academy of Sciences of the United States of America, vol. 100, no. 2, pp. 521-526, 2003.

[42] C. Yang, M. Cao, Y. Liu et al., "Inhibition of cell invasion and migration by CEACAM1-4S in breast cancer," Oncology Letters, vol. 14, no. 4, pp. 4758-4766, 2017. 


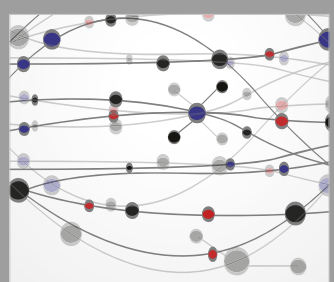

The Scientific World Journal
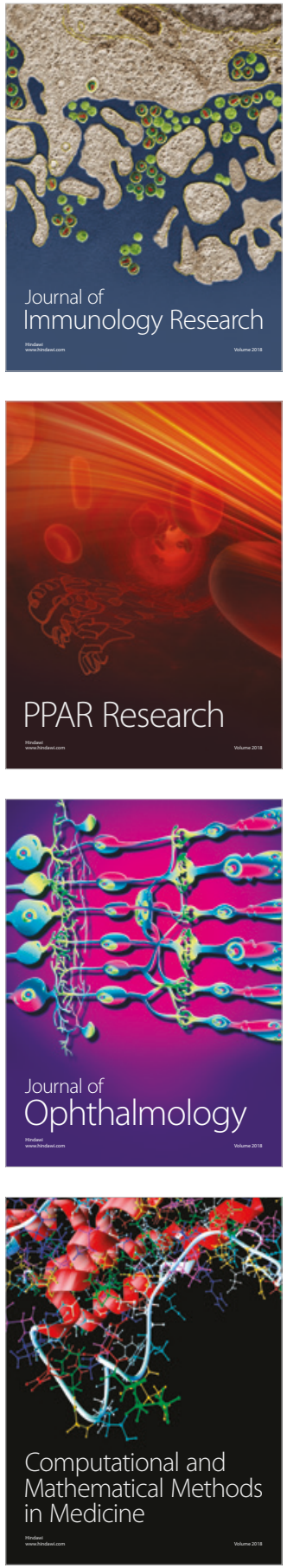

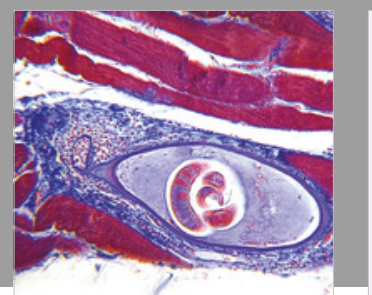

Gastroenterology Research and Practice

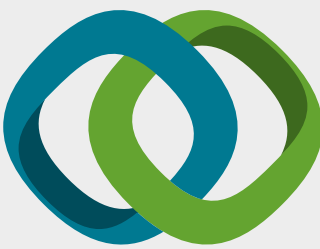

\section{Hindawi}

Submit your manuscripts at

www.hindawi.com
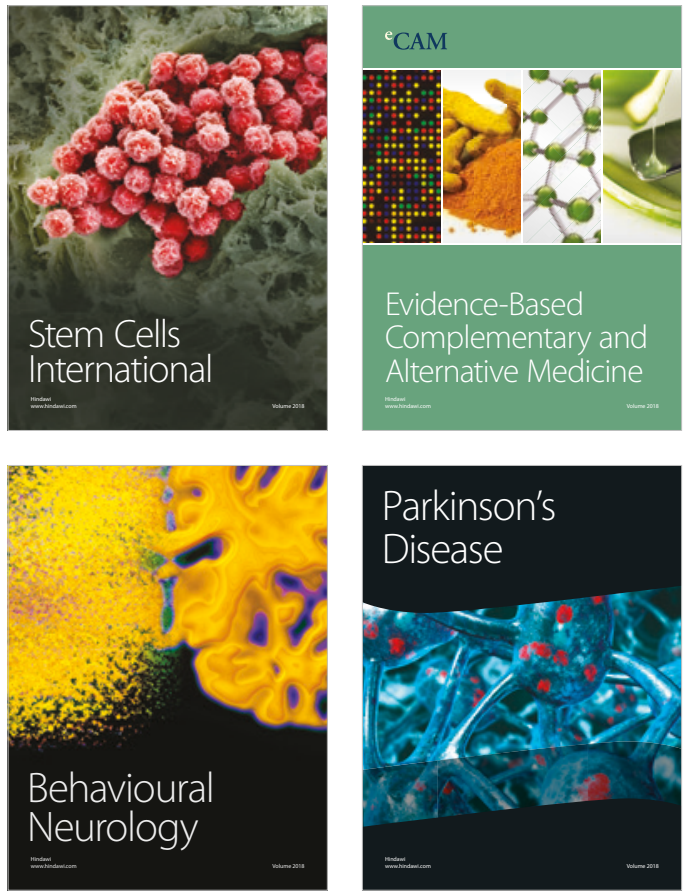

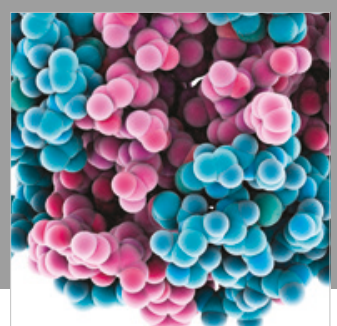

ournal of

Diabetes Research

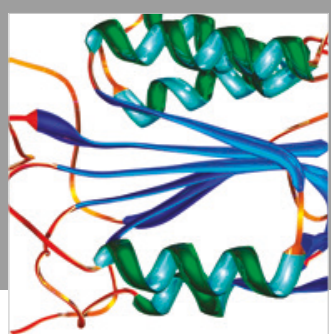

Disease Markers
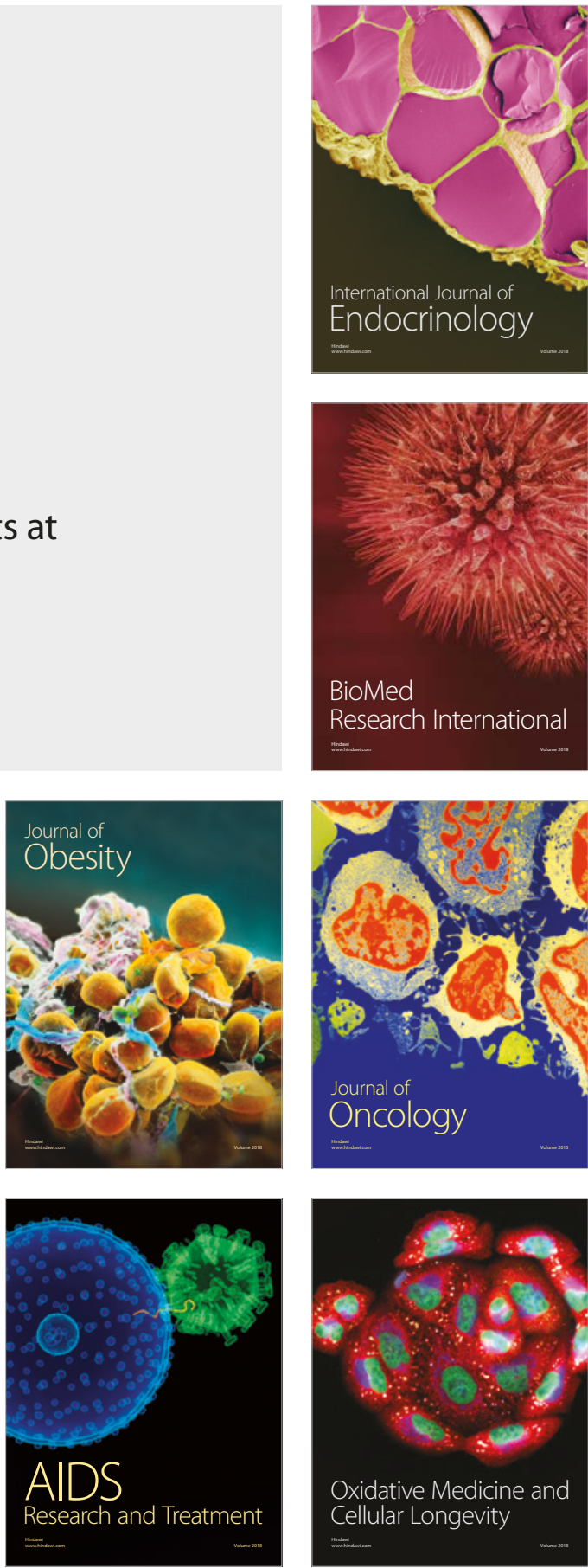